\title{
Secondary Neurulation Defects-1 : Retained Medullary Cord
}

\author{
Kyung Hyun Kim, ${ }^{1}$ Ji Yeoun Lee, ${ }^{1,2}$ Kyu-Chang Wang ${ }^{1}$ \\ Division of Pediatric Neurosurgery, Seoul National University Children's Hospital, Seoul, Korea \\ Department of Anatomy, ${ }^{2}$ Seoul National University College of Medicine, Seoul, Korea
}

Retained medullary cord (RMC) is a relatively recent term. Pang et al. newly defined the RMC as a late arrest of secondary neurulation leaving a non-functional vestigial portion at the tip of the conus medullaris. RMC, which belongs to the category of closed spinal dysraphism, is a cord-like structure that is elongated from the conus toward the cul-de-sac. Because intraoperative electrophysiological confirmation of a non-functional conus is essential for the diagnosis of RMC, only a tentative or an assumptive diagnosis is possible before surgery or in cases of limited surgical exposure. We suggest the term 'possible RMC' for these cases. An RMC may cause tethered cord syndrome and thus requires surgery. This article reviews the literature to elucidate the pathoembryogenesis, clinical significance and treatment of RMCs.

Key Words : Secondary neurulation · Conus medullaris · Intraoperative electrophysiological monitoring · Closed spinal dysraphism - Tethered cord syndrome.

\section{INTRODUCTION}

The lower part of the spinal cord is known to be formed through secondary neurulation from the caudal cell mass. In humans, it is thought that primary neurulation generates the spinal cord down to the S1-S2 vertebral body junction, below which structures including the sacrococcygeal spine, conus medullaris, and filum terminale are known to be made by secondary neurulation ${ }^{9,10,14)}$. The conus medullaris and filum terminale are formed by a degeneration process during the development of the caudal part of the spinal cord. A retained medullary cord (RMC) occurs as a result of the failure of this regression process.

$\mathrm{RMC}$ is a relatively recent term. Previously, these lesions were called 'low-lying conus' or 'giant cyst of the filum terminale', although the meaning of these terms is not exactly the same as RMC. Pang et al. ${ }^{16)}$ newly defined the RMC as a late arrest of secondary neurulation.

Although RMCs are known to be rare, they are often encountered in the clinic. It is thought that the number of RMC patients tends to be underreported because RMCs have been newly defined. In 2019, among 74 patients who underwent surgery for spinal dysraphism, six patients had RMCs and additional four were diagnosed as 'possible RMC' in the Seoul National University Children's Hospital (SNUCH). We think that quite a few RMCs have been previously classified as 'lowlying conus with or without terminal syrinx or caudal lipoma'. It is expected that RMCs are not very rare, as their classifica-

\footnotetext{
- Received : February 26, 2020 •Revised : March 4, 2020 •Accepted : March 13, 2020

- Address for reprints : Kyu-Chang Wang

Division of Pediatric Neurosurgery, Seoul National University Children's Hospital, 101 Daehak-ro, Jongno-gu, Seoul 03080, Korea Tel : +82-2-2072-3489, Fax : +82-2-2072-0274, E-mail : kcwang@snu.ac.kr, ORCID : https://orcid.org/0000-0001-7440-6650
}

This is an Open Access article distributed under the terms of the Creative Commons Attribution Non-Commercial License (http://creativecommons.org/licenses/by-nc/4.0) which permits unrestricted non-commercial use, distribution, and reproduction in any medium, provided the original work is properly cited. 
tion has become more widely known.

This article reviews the literature on the pathoembryogenesis, clinical significance and treatment of RMCs.

\section{PATHOEMBRYOGENESIS AND DEFINITION}

An RMC is a robust, elongated spinal-cord-like structure that continues to the cul-de-sac ${ }^{16)}$. Simply, an RMC looks like a 'low-lying conus', but it has a non-functioning component. Because the non-functional conus is indistinguishable from a functioning conus on neuroimaging, confirming the presence or absence of a non-functional conus with intraoperative neurophysiological monitoring (IONM) is essential for the diagnosis of RMC.

RMC is caused by the complete or partial arrest of secondary neurulation $^{16)}$. It is not yet clear why arrest occurs, but it is thought that apoptosis plays a major role ${ }^{16)}$. Animal studies have provided some evidence, and numerous apoptosis-related substances have been found in late secondary neural tube formation $^{1-7,12,13,18,20,21)}$.

The secondary neural tube transiently has a lumen. Therefore, when the arrest of regression occurs rather early, this may result in the 'low-lying conus with distal luminal dilatation'. Although it was not included in the original description of an RMC by Pang et al. ${ }^{16)}$ in 2011, we believe that it is reasonable to include this cystic variant, with at least some lesions called 'terminal syrinx with low-lying conus' or 'giant filar cysts', in the category of RMC (Fig. 1).

In a broader sense, the common entity of a filar cyst and the rarer entity of a terminal myelocystocele (TMCC) are also remnants of the regressive phase of secondary neurulation at different time points (filar cyst : later, TMCC : earlier) and may be included in the category of RMC (retainment of the medullary cord, which represents the secondary neural tube before regression). However, because its clinical manifestation and significance are different from those of 'RMC in a strict sense', we do not recommend to include filar cyst and TMCC in the category of RMC for practical reasons.

Some RMC cases have varying amounts of fat in the low-lying conus, which needs to be differentiated from some types of lumbosacral lipomatous malformation. Filar and caudal lipomas are believed to be an aberrant differentiation of the caudal cell mass rather than a failure of regression. In cases of filar lipoma, the end of the conus is well defined at the cranial side of the lipoma. In caudal lipoma, it is common that the ta-
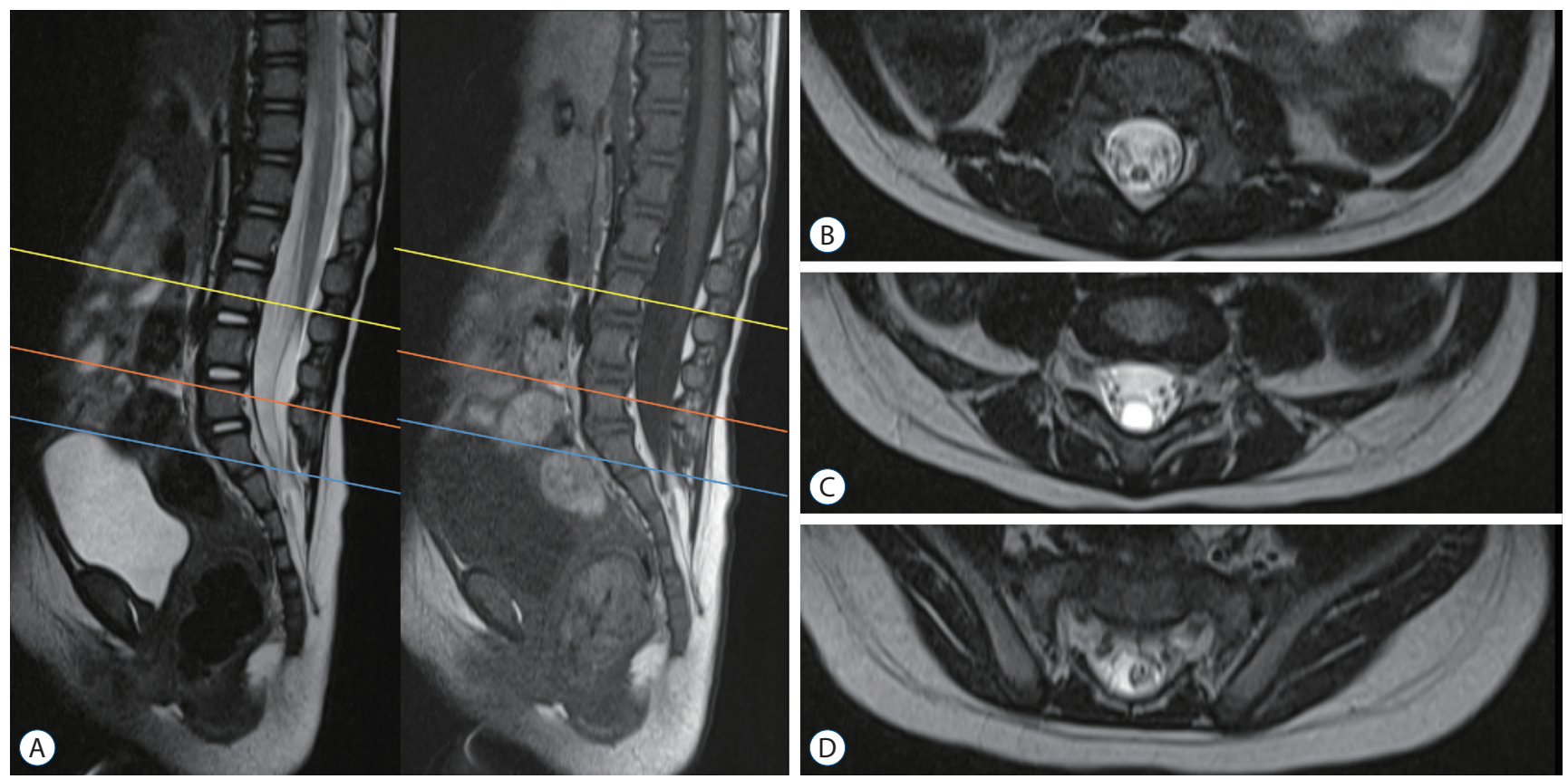

Fig. 1. Magnetic resonance imaging of a patient with 'terminal syrinx with low-lying conus'. A : This demonstrates that the thick medullary cord extends down to the lower lumbar area, where it ends with a cyst that attaches to the cul-de-sac. B-D : They show T2 axial images at L3 (yellow line in A), L5 (orange line), and S1-2 (blue line), respectively. 
pered conus is continuous with the distally enlarging lipoma. The tip of the conus is functional, and the level of the upper cut for untethering is at the upper part of the lipoma and not at the conus. If fatty tissue and a non-functional cord are included in the tapering conus, it is considered an RMC, as was the case in patient 3 in the first description of an RMC by Pang et al. ${ }^{16)}$ However, cases of gray zone between the caudal lipoma and RMC are present on neuroimaging : there was no tapering or enlargement of the conus with the gradual transition to a fat-containing mass at the end (Fig. 2B). We think that even without distal tapering, a low-lying conus with various amounts of fat can be included in the definition of RMC if there is a non-functional part in the conus. In these cases, operative exposure with IONM of the critical region is necessary for the definitive diagnosis.

The original definition of an RMC can be applied to lesions that continue to the cul-de-sac ${ }^{16)}$ (Fig. 2A). However, we do not know the threshold (criteria) of 'low-lying conus' on possible presence of a non-functional conus. The conus with arrested regression may be located at more cranial part than the end of dural sac. The end of the RMC was located at L4, not at the cul-de-sac, in patient 2 in the first description of RMC by Pang et al. ${ }^{16)}$ If the modified definition of RMC includes any lesion in which the tip of the conus is located above the culde-sac with an identifiable filum (which may be thick) at the distal part of the conus, one more issue is the surgical identification of a non-functional distal cord as a diagnostic criterion. This point differentiates an RMC from a 'simply low-lying conus', which has a functioning end. Often, sectioning of the fi- lum through the limited exposure of the dura is enough to release the tethered cord if the filum (which may be thick) is formed at the caudal end. In these cases, as the conus is not exposed, it is not possible to electrophysiologically or histologically confirm whether the low-lying conus contains the non-functional vestigial part. For these cases, only an assumptive diagnosis is possible, and we suggest the term 'possible RMC'. The fact that the filum normally includes neural components makes this issue more difficult when only a small part of the presumed RMC-filum transition is resected.

Currently, the SNUCH team uses the term 'possible RMC' for lesions with a low-lying conus but with no electrophysiological or histological confirmation of the presence of a nonfunctional conus before surgery or in cases of limited surgical exposure. We agree that there may be overuse or abuse of the term 'possible RMC'. We expect a clear definition of this issue.

\section{CLINICAL MANIFESTATIONS}

An RMC may show all the cutaneous markers (crater, hypertrichosis, hemangioma, skin tag, dimple, etc.) seen in spinal dysraphism patients. The associated diseases include caudal agenesis, presacral cysts, hindgut abnormalities, caudal lipomas, and various anorectal and genitourinary anomalies, all of which are related to secondary neurulation ${ }^{15,16)}$. The clinical symptoms may include those found in tethered cord syndrome, such as progressive foot deformities, neurogenic bladder, incontinence, frequent urinary tract infections, urodynamic abnor-
(A)

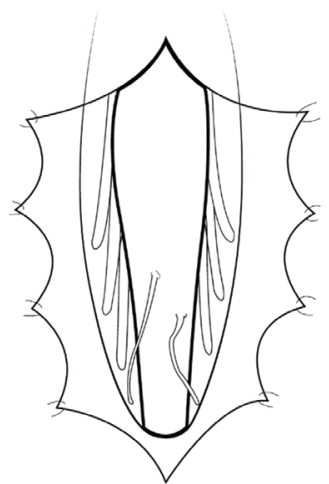

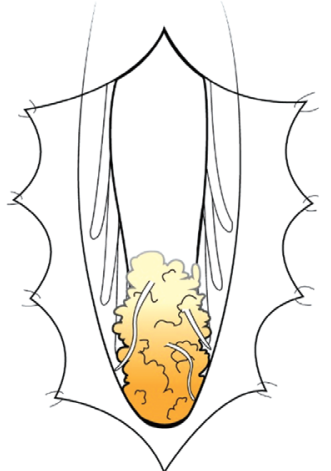

(C)

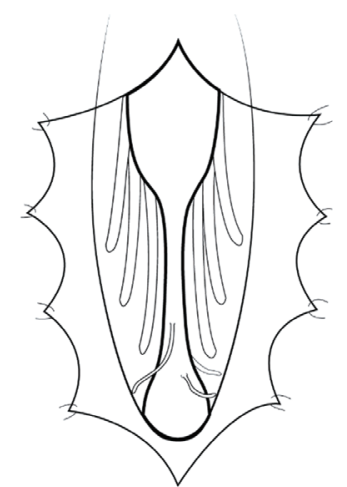

Fig. 2. Schematic illustrations of three types of non-cystic RMCs. Fine vestigial nerve twigs emanate from the medullary cord. A : It shows a typical RMC. $\mathrm{B}$ : It reveals no terminal tapering but enlargement of the conus with gradual transition to a fat-containing mass at the end. The 'cord portion' has a nonfunctional part in the RMC, which is different from a simple caudal lipoma. C : It shows an hourglass-shaped RMC with an intervening filum-like structure between the conus and RMC. If the part distal to the filum-like structure is functional but not electrophysiologically connected to the 'upper cord', it is considered a junctional neural tube defect. RMC : retained medullary cord. 
malities (diminished bladder capacity or detrusor-sphincter dyssynergia), dysesthesia, leg pain, and coccydynia ${ }^{15,16)}$. Neurological deficits may or may not be present. In symptomatic cases, surgery needs to be performed to relieve the tethering of the cord. Pang et al ${ }^{15,16)}$ recommended prophylactic surgery, even in the absence of any symptoms.

\section{NEUROIMAGING}

Upon magnetic resonance imaging (MRI) of an RMC, the conus appears in the lower position due to the tapering from the conus to the medullary cord without any interruption or visual clues ${ }^{15)}$. In some cases, a thick filum is located between the conus and the medullary cord, resulting in an hourglass shape that narrows at the filum and then widens again at the medullary $\operatorname{cord}^{15)}$ (Fig. 2C). In certain cases, the medullary

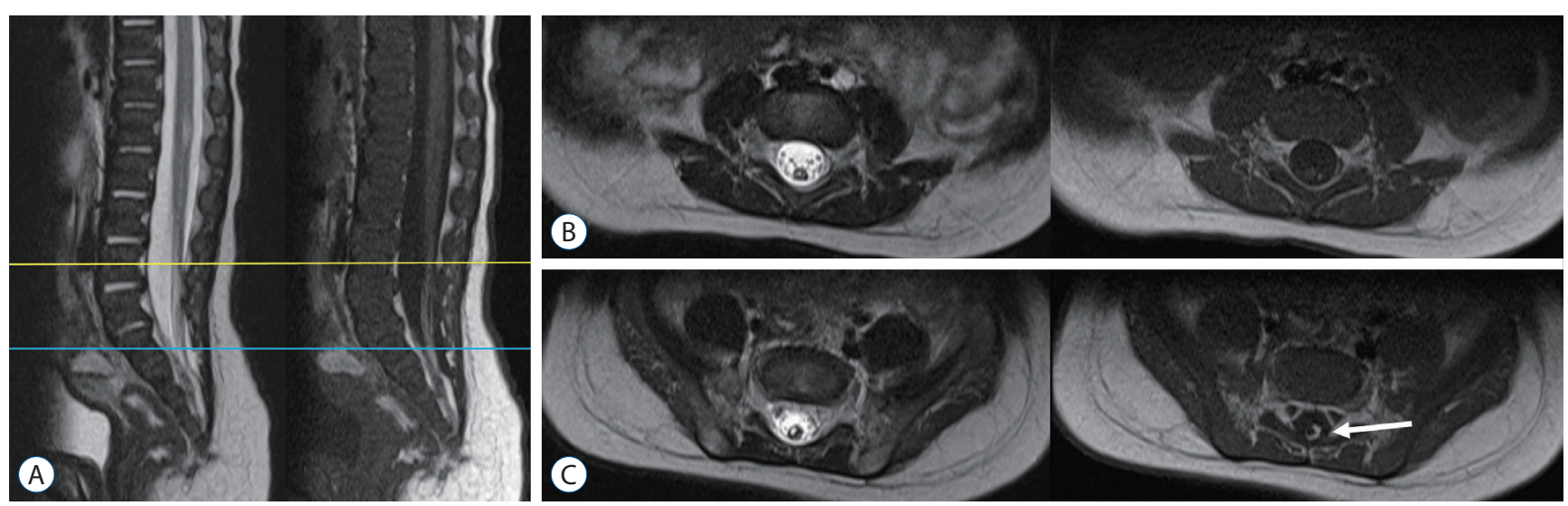

Fig. 3. MRI of a 'possible retained medullary cord' patient. A : The medullary cord partially contains fat tissue, resulting in high signal intensity in the T1weighted MR image (arrow in C). In this case, only a small segment of the distal part was resected through a limited exposure of the lipoma. Exploration of the whole conus area, which may include the non-functional parts, was not performed. B and C: They show axial images at L4 (yellow line in A) and S1 (blue line), respectively. MRI : magnetic resonance imaging.
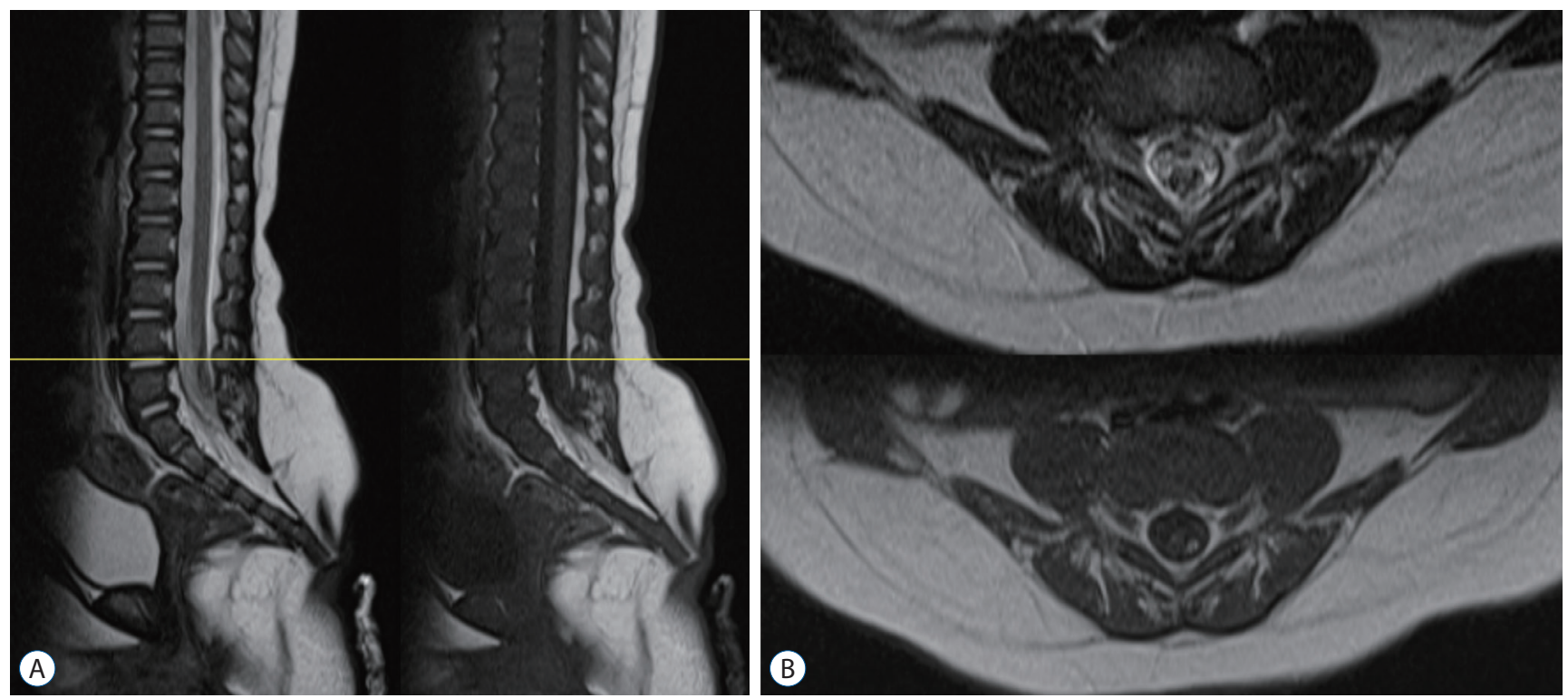

Fig. 4. MRI of an RMC patient. A : The signal intensity is indistinguishable between the spinal cord and the RMC. On operation, the non-functional part of the RMC was identified by electrophysiological monitoring and was resected. B : Axial images at L4-5 (yellow line in A). MRI : magnetic resonance imaging, RMC : retained medullary cord. 
cord may partially contain fat tissue, resulting in high signal intensity on T1-weighted MR images ${ }^{15)}$ (Fig. 3). In most cases, however, the signal intensity is indistinguishable between the spinal cord and the RMC (Fig. 4).

An RMC can, by default, be accompanied by other secondary neurulation anomalies such as presacral cysts or sacral agenesis. In the literature, caudal lipomas, limited dorsal myeloschisis, congenital dermal sinuses, subcutaneous meningoceles, and split cord malformations can be associated with $\mathrm{RMCs}^{8,11,16,19)}$.
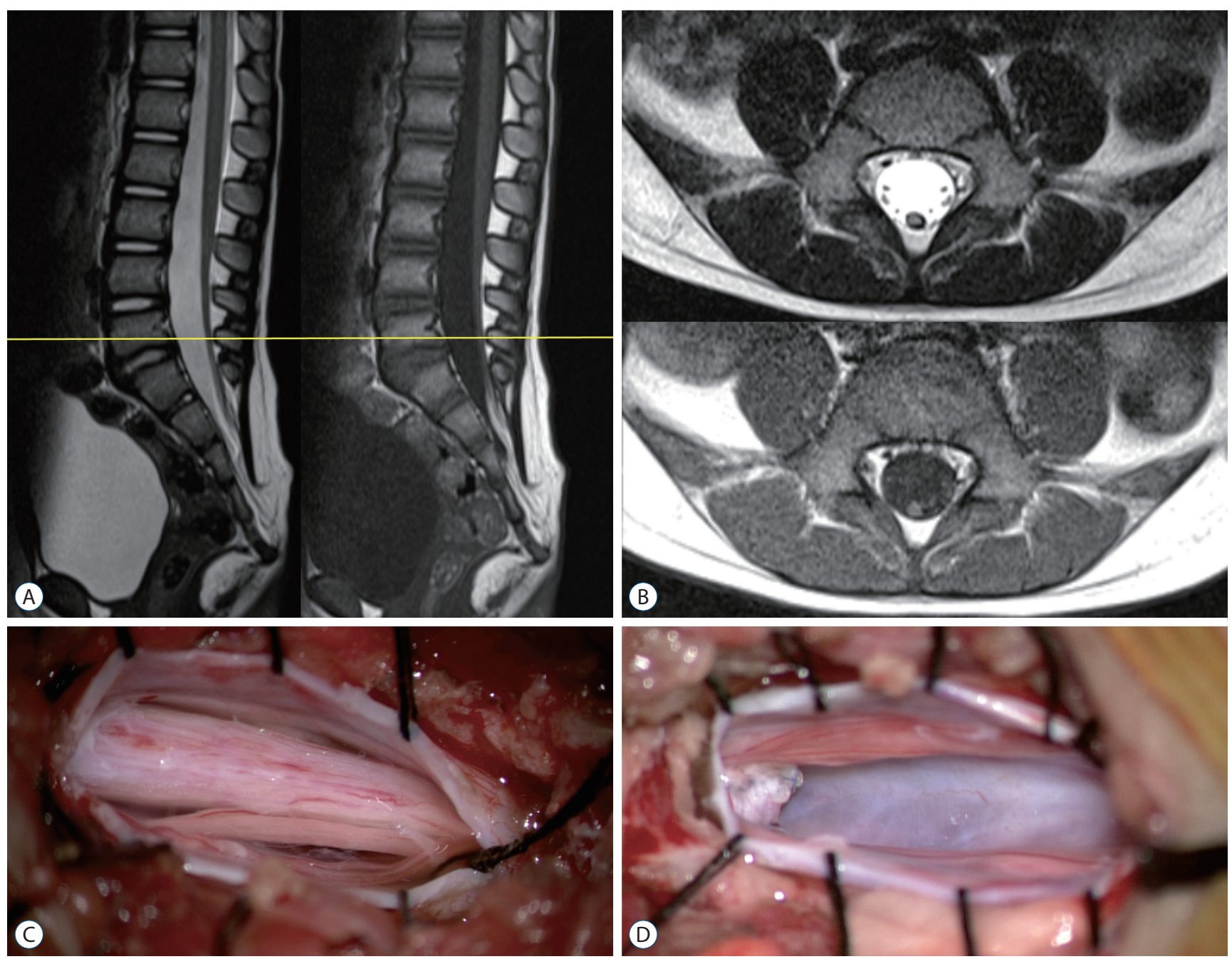

Fig. 5. Magnetic resonance imaging and intraoperative photograph of a retained medullary cord patient. A : A cord-like structure extending to the sacral area. B : Axial images at L5 (yellow line in A). C : The limited L5 unilateral partial hemilaminectomy shows a thick, pia-covered medullary cord passing through. D : After resection of the non-functional medullary cord. Pial reconstruction was performed at the most caudal part of the cord. Removal of the non-functional segment creates a gap and may reduce the chance of retethering compared with just cutting. 
other hand, from a practical point of view, if the goal is only untethering, it may be sufficient to simply cut the tethered structures through a limited exposure ${ }^{15)}$ (Fig. 5). Sala et al. ${ }^{17)}$ suggested rostrocaudal stimulation. This is a method to find the exact point when a signal becomes null. When severing, it may be convenient to cut the safe caudal end first and then gently lift the cord to find the junction site between the functional and non-functional parts by stimulating the electrical signal while being careful not to spread the signal through the cerebrospinal fluid (CSF). At this time, if a cottonoid is placed under the cord, it can easily prevent electric signals from spreading through the CSF.

When performing stimulation, it is important to ensure that the stimulating probe is working properly and to exclude false negatives. In addition, to prevent retethering, it is advisable to reconstruct the pia layer. The knot should be placed inward toward the end of the conus.

\section{HISTOLOGY}

The characteristic histological findings of RMCs include a dense glioneuronal core surrounded by variable amounts of fibrous and adipose tissues, an ependymal-lined central cavity and vestigial nerve roots ${ }^{15,16}$. There are fine nerve twigs of nerve roots that emanate from the medullary cord. However, these are vestigial nerve roots that are not functional ${ }^{16)}$. These are mostly randomly positioned or exhibit unusual anatomical courses, unlike functional roots. While the caudal end of the spinal cord shows meandering surface vascular structures, RMCs may exhibit relatively straight blood vessels.

\section{CLINICAL OUTCOME}

The prognosis of RMCs seems to be good. No patients have demonstrated new neurological deficits or progression after surgery in the literature ${ }^{8,11,15-17,19)}$. Since there is not much information available on RMC, it will be meaningful to compare the clinical outcomes of wide exposure with excision of the whole non-functional cord and those of limited exposure with only untethering.

\section{CONCLUSION}

Almost a decade has passed since the new concept of RMC was proposed by Pang et al. ${ }^{16)}$ Although it is commonly accepted that RMCs are caused by the late arrest of secondary neurulation, some issues remain to be solved, particularly in terms of the definition and nomenclature. It is important to consider other disease entities that may be associated with secondary neural tube formation when treating RMCs. The goal of RMC surgery should be untethering. However, an RMC is indistinguishable from the normal spinal cord with MR imaging as well as with the naked eye. Therefore, it is important to use IONM to identify the correct area to cut, where no signal emerges.

\section{CONFLICTS OF INTEREST}

No potential conflict of interest relevant to this article was reported.

\section{INFORMED CONSENT}

This type of study does not require informed consent.

\section{AUTHOR CONTRIBUTIONS}

\author{
Conceptualization : KCW \\ Data curation : KHK, KCW \\ Formal analysis : KHK, KCW \\ Funding acquisition : JYL, KCW \\ Methodology : KHK, JYL, KCW \\ Project administration : JYL, KCW \\ Visualization : KHK, KCW \\ Writing - original draft : KHK, KCW \\ Writing - review \& editing : JYL, KCW
}

\section{ORCID}

Kyung Hyun Kim https://orcid.org/0000-0002-8238-2043

Ji Yeoun Lee https://orcid.org/0000-0003-0464-7605 
Kyu-Chang Wang https://orcid.org/0000-0001-7440-6650

\section{- Acknowledgements}

The authors acknowledge Mr. Jae Jeong Ro for the preparation of the medical illustrations.

This work was supported by the National Research Foundation of Korea (NRF) Grant funded by the Korean Government (MSIP) (2018R1A5A2025964). This study was also supported by grant No. 04-2017-0240 from the Seoul National University Hospital Research Fund.

\section{References}

1. Borner C, Monney L, Olivier R, Rossé T, Häcki J, Conus S : Life and death in a medieval atmosphere. Cell Death Differ 6 : 201-206, 1999

2. Chung YN, Lee DH, Yang HJ, Kim SK, Lee YJ, Lee MS, et al. : Expression of neuronal markers in the secondary neurulation of chick embryos.

Childs Nerv Syst 24 : 105-110, 2008

3. Elmore S: Apoptosis: a review of programmed cell death. Toxicol Pathol 35 : 495-516, 2007

4. Griffith CM, Wiley MJ, Sanders EJ : The vertebrate tail bud: three germ layers from one tissue. Anat Embryol (Berl) 185 : 101-113, 1992

5. Hetts SW : To die or not to die: an overview of apoptosis and its role in disease. JAMA 279 : 300-307, 1998

6. Hughes AF, Freeman RB : Comparative remarks on the development of the tail cord among higher vertebrates. J Embryol Exp Morphol 32 : 355-363, 1974

7. Mills $C L$, Bellairs $R$ : Mitosis and cell death in the tail of the chick embryo. Anat Embryol (Berl) 180 : 301-308, 1989

8. Morioka T, Murakami N, Kanata A, Tsukamoto H, Suzuki SO : Retained medullary cord with sacral subcutaneous meningocele and congenital dermal sinus. Childs Nerv Syst 36 : 423-427, 2020

9. Müller F, O'Rahilly $R$ : The development of the human brain, the closure of the caudal neuropore, and the beginning of secondary neurulation at stage 12. Anat Embryol (Berl) 176 : 413-430, 1987

10. Müller F, O'Rahilly $R$ : The primitive streak, the caudal eminence and related structures in staged human embryos. Cells Tissues Organs $177: 2-20$, 2004

11. Murakami N, Morioka T, Shimogawa T, Hashiguchi K, Mukae N, Uchihashi $K$, et al. : Retained medullary cord extending to a sacral subcutaneous meningocele. Childs Nerv Syst 34 : 527-533, 2018

12. Nievelstein RA, Hartwig NG, Vermeij-Keers C, Valk J : Embryonic development of the mammalian caudal neural tube. Teratology $48: 21-31$, 1993

13. Nijhawan $D$, Honarpour $N$, Wang $X$ : Apoptosis in neural development and disease. Annu Rev Neurosci 23 : 73-87, 2000

14. O'Rahilly R, Müller $F$ : Neurulation in the normal human embryo. Ciba Found Symp 181 : 70-82; discussion 82-89, 1994

15. Pang D, Chong S, Wang KC : Secondary Neurulation Defects-1: Thickened Filum Terminale, Retained Medullary Cord in Di Rocco C, Pang D, Rutka J (eds) : Textbook of Pediatric Neurosurgery. Cham : Springer, 2017, pp1-18

16. Pang D, Zovickian J, Moes GS : Retained medullary cord in humans: late arrest of secondary neurulation. Neurosurgery 68 : 1500-1519; discussion 1519, 2011

17. Sala F, Barone G, Tramontano V, Gallo P, Ghimenton C : Retained medullary cord confirmed by intraoperative neurophysiological mapping. Childs Nerv Syst 30 : 1287-1291, 2014

18. Schoenwolf GC: Morphogenetic processes involved in the remodeling of the tail region of the chick embryo. Anat Embryol (Berl) 162 : 183197, 1981

19. Shirozu N, Morioka T, Inoha S, Imamoto N, Sasaguri T : Enlargement of sacral subcutaneous meningocele associated with retained medullary cord. Childs Nerv Syst 34 : 1785-1790, 2018

20. Yang HJ, Wang KC, Chi JG, Lee MS, Lee YJ, Kim SK, et al. : Cytokinetics of secondary neurulation in chick embryos: Hamburger and Hamilton stages 16-45. Childs Nerv Syst 22 : 567-571, 2006

21. Yang HJ, Wang KC, Chi JG, Lee MS, Lee YJ, Kim SK, et al. : Neural differentiation of caudal cell mass (secondary neurulation) in chick embryos: Hamburger and Hamilton stages 16-45. Brain Res Dev Brain Res 142 : 31-36, 2003 